\title{
Preferential Sputtering induced Cr-Diffusion during Plasma Exposure of WCrY Smart Alloys
}

\author{
J. Schmitz ${ }^{\mathrm{a}, \mathrm{b}, *}$, A. Mutzke ${ }^{\mathrm{c}}$, A. Litnovsky ${ }^{\mathrm{a}}$, F. Klein ${ }^{\mathrm{a}}$, X.Y. Tan ${ }^{\mathrm{a}, \mathrm{d}}$, T. Wegener ${ }^{\mathrm{a}}$, P. Hansen ${ }^{\mathrm{a}}$, A. Eksaeva ${ }^{\mathrm{a}}$, M. \\ Rasinski $^{\mathrm{a}}$, A. Kreter ${ }^{\mathrm{a}}$, J. Gonzalez-Julian ${ }^{\mathrm{a}}$, J.W. Coenen ${ }^{\mathrm{a}}$, Ch. Linsmeier ${ }^{\mathrm{a}}$, M. Bram ${ }^{\mathrm{a}}$ \\ ${ }^{a}$ Forschungszentrum Jülich GmbH, Institut für Energie- und Klimaforschung, 52425 Jülich, Germany \\ ${ }^{b}$ Department of Applied Physics, Ghent University, 9000 Ghent, Belgium \\ ${ }^{c}$ Max-Planck-Institute for Plasmaphysics, Wendelsteinstrasse 1, 17491 Greifswald, Germany \\ ${ }^{d}$ School of Materials Science and Engineering, Hefei University of Technology, Hefei 230009, China
}

\begin{abstract}
WCrY Smart Alloys are developed as first wall material of future fusion devices such as DEMO. They aim at behaving like pure $\mathrm{W}$ during plasma operation due to depletion of the alloying elements $\mathrm{Cr}$ and $\mathrm{Y}$. The $\mathrm{Cr}$ concentration gradients induced by preferential plasma sputtering cause $\mathrm{Cr}$-diffusion. The exposure of $\mathrm{WCrY}$ and $\mathrm{W}$ samples to pure D plasma, with a plasma ion energy of $220 \mathrm{eV}$, is simulated using the dynamic version of SDTrimSP. Cr-diffusion is included into the model. Simulation results are compared with experimental results. At sample temperatures of more than $600{ }^{\circ} \mathrm{C}$ and sputtering by D plus residual oxygen in the plasma ion flux, the Cr-transport to the surface leads to enhanced erosion for WCrY samples. A diffusion coefficient for Cr in WCrY of the order of $1 \cdot 10^{-17} \mathrm{~m}^{2} / \mathrm{s}$ is determined. The suitability of $\mathrm{WCrY}$ as first wall armour and the influence of further effects, considering especially Cr-diffusion, is discussed.
\end{abstract}

Keywords: smart alloys, plasma-wall-interaction, plasma ion irradiation, preferential sputtering, DEMO, modelling, diffusion, SDTrimSP

\section{Introduction}

Plasma-facing components (PFCs) of future fusion devices have to fulfill various requirements, which are described for example in [20]. Tungsten (W) is cur5 rently the preferred first wall material for the demonstration power plant DEMO. This material possesses various advantageous properties such as a very high melting point of more than $3300^{\circ} \mathrm{C}$ and low erosion yields [3]. Yet, there are also some drawbacks regarding the usage 10 of pure W: besides its inherent brittleness at room temperature, $\mathrm{W}$ oxidises rapidly at temperatures of above $700^{\circ} \mathrm{C}$, above $900^{\circ} \mathrm{C}$ sublimation of the oxide becomes significant [19]. This makes pure $\mathrm{W}$ a potential safety hazard if a Loss-Of-Coolant-Accident (LOCA) with ad15 ditional air ingress occurs. In that case the cooling system fails and the wall temperatures rise from $1000^{\circ} \mathrm{C}$ to $1200^{\circ} \mathrm{C}$ for a few days to several months, depending on the blanket design, due to nuclear decay heat [22].

\footnotetext{
* Corresponding author

Email address: jan.schmitz@fz-juelich.de (J. Schmitz)
}

$\mathrm{W}$, having been activated by neutron irradiation during 20 plasma operation, then forms radioactive $\mathrm{WO}_{3}$. Sublimation of $\mathrm{WO}_{3}$ leads to its mobilisation and thus the possible release of radioactive material out of the vacuum vessel. This has to be prevented to build safe fusion reactors.

25 Smart WCrY alloys aim at suppressing the oxidation of $\mathrm{W}$ during LOCA. These alloys contain, besides $\mathrm{W}$, mainly chromium $(\mathrm{Cr})$ and also small amounts of yttrium (Y). Their smartness consists in their ability to adapt to two kind of reactor scenarios: on the one hand, the lighter alloying elements $\mathrm{Cr}$ and $\mathrm{Y}$ are depleted towards the surface due to preferential sputtering during regular plasma operation. This leaves the plasma facing a pure $\mathrm{W}$ surface. In that way the material benefits from the low erosion yields of $\mathrm{W}$. On the other hand, in case of LOCA with oxygen-containing atmosphere, the alloying elements diffuse towards the alloy's surface. A protective $\mathrm{Cr}_{2} \mathrm{O}_{3}$ scale forms and hence the formation of $\mathrm{WO}_{3}$ is suppressed. The optimum composition for WCrY thin film systems was found to be ${ }_{40} \mathrm{~W}-11.4 \mathrm{wt} \% \mathrm{Cr}-0.6 \mathrm{wt} \% \mathrm{Y}$, these are described in detail 
in [36] and [21]. They demonstrated a significant oxidation suppression so that it was decided to produce bulk WCrY samples with the same composition. Bulk WCrY samples with the optimum composition achieved an ox-

45 idation suppression of more than three orders of magnitude in comparison to pure $\mathrm{W}$ samples of the same geometry. The oxidation rate was measured as gradient of mass change per area during oxidation in dry air at 100 $1000^{\circ} \mathrm{C}[29]$.

50 Given a convincing oxidation performance, the plasma performance of the WCrY smart alloys has to be evaluated. The alloys are developed for the first wall of DEMO. To choose relevant experimental conditions at 105 first possible plasma conditions for the DEMO first wall ${ }_{55}$ are investigated: In [10] it is stated that a pulsed baseline DEMO reactor concept is prioritised with the possibility to be upgraded to a long-pulse or steady-state machine. The European DEMO design described in 110 [38] assumes a peak heat flux of maximum $1 \mathrm{MW} \mathrm{m}^{-2}$

60 for the first wall. The first wall surface temperature during regular operation is restricted by the temperature limit of the considered structural material. The reduced activation ferritic martensitic steel EUROFER97 [37] with a temperature limit of $550{ }^{\circ} \mathrm{C}$ [37] is one 65 option considered for the European DEMO. The thermal conductivity of $\mathrm{W}$ is about $1 \cdot 10^{2} \mathrm{Wm}^{-1} \mathrm{~K}^{-1}$ and tends to decrease under fusion-relevant radiation conditions [4]. [2] found a value of about $5 \cdot 10^{1} \mathrm{Wm}^{-1} \mathrm{~K}^{-1}$ at temperatures between $500^{\circ} \mathrm{C}$ and $700{ }^{\circ} \mathrm{C}$ for bulk

70 WCrY samples with compositions similar to the one of the samples used for this work, which means a decrease in the thermal conductivity by a factor of two in comparison to pure $\mathrm{W}$. Considering a $3 \mathrm{~mm}$ thick $\mathrm{W}$ or WCrY armour layer, first wall temperatures should 75 not largely exceed $600^{\circ} \mathrm{C}$. Besides heat loads, particle loads of different origin strike the wall and can cause sputtering: plasma ions may be accelerated to energies exceeding the sputter thresholds, additional erosion is caused by charge exchange (CX) neutrals. In 80 future fusion devices the erosion due to energetic neutrals is assumed to be of the same order of magnitude as for ions [27]. In some DEMO wall areas their contribution even dominates [34]. The thermal ions and other particles have a continuous energy distribution. 85 Some particle energies lie above the sputter threshold, while the average stays below it. Temperatures in the DEMO Scrape-Off-Layer (SOL) are expected to be below $100 \mathrm{eV}$ according to [37], whereas [14] expects more than $100 \mathrm{eV}$. The design question whether high

90 heat flux limiters will be installed still remains open for DEMO [37]. When this limited configuration is used, e.g. during ramp-up or ramp-down of the plasma, the plasma gets closer to the wall and ion energies are elevated. Extrinsic impurity seeding can be used to reduce 5 heat fluxes on PFCs. Concentrations of less than $1 \%$ of argon (Ar) and xenon (Xe) or nitrogen (Ni) are foreseen for DEMO [16]. Moreover helium (He) produced by the deuterium-tritium (DT) reaction will always be present in the plasma. The heavy impurity species sputter $\mathrm{W}$ more effectively than the light hydrogen fuel ions.

In [30] it is stated that diffusion of $\mathrm{Cr}$ towards the surface is a determining factor for the erosion of WCrY at higher ion energies. Similarly, studies on W bombarded by energetic carbon (C) impurity ions presented in [31] underline the importance of thermally activated processes such as diffusion and segregation for the erosion of mixed materials. Therefore, in this publication we focus on modelling the plasma impact onto the alloy's surface composition including thermal diffusion. The effects of D ion irradiation, oxygen plasma content and Cr-diffusion are examined. Results obtained with the Monte-Carlo code SDTrimSP [24] are compared to experimental results.

\section{Experimental}

\subsection{Plasma exposure}

\subsubsection{Description}

The exposure to steady-state plasma took place in the linear plasma device PSI-2 [18]. In PSI-2 sputtering of a material is induced by bombardment with energetic ions. In contrast to the thermal particle energy distribution in DEMO, the ions during plasma exposure in PSI2 are close to mono-energetic as determined by the applied bias voltage. Hence a DEMO-relevant ion energy has to be chosen for experiments. The PSI-2 plasma source was operated with pure D as working gas. However, a minute residual oxygen amount is present on Quadrupole Mass Spectrometry (QMS) data recorded in the PSI-2 vacuum vessel. The line radiation of the impurity oxygen was too weak to be detected with emission spectroscopy measurements, similar to the spectroscopy measurements described in [5] for an oxygen content of $0.2 \%$ in PISCES-B. We therefore assume the oxygen impurity content to be of similar order as presented in [5]. In pure D plasma, no W sputtering is expected for ion energies of around $100 \mathrm{eV}$, as the sputter threshold for $\mathrm{D}$ on $\mathrm{W}$ is around $200 \mathrm{eV}$. For instance in [7] an experimental value of $178 \mathrm{eV}$ as well as a fitted value of $201 \mathrm{eV}$ are given. The $\mathrm{W}$ sputter threshold for residual oxygen in the plasma is considerably lower, in [7] the calculated threshold is $42 \mathrm{eV}$. To have $\mathrm{W}$ erosion caused by $\mathrm{D}$ sputtering and not only residual $\mathrm{O}$, an ion 
energy of $220 \mathrm{eV}$ was chosen. The threshold for $\mathrm{T}$ on $\mathrm{W}$ is around $130 \mathrm{eV}$ according to [7] and thus significantly lower in a D+T plasma than for pure D. As wall temDEMO first wall should not be much higher than $600{ }^{\circ} \mathrm{C}$ (see chapter 1 ), the sample temperature during the PSI- 2 experiments should also be in this range. The temperature of all sample surfaces is monitored during the exposure using an infra-red camera.

150 For the experiment two WCrY samples, called WCrY1 and WCrY2, and two W samples manufactured according to ITER specification [15], W1 and W2, were exposed simultaneously on the same target holder. WCrY samples had the optimum composition (see chapter 1) 155 and were manufactured according to the procedure described in [30]. The four WCrY and W samples were all prepared in the same manner: after wire erosion cutting they were manually ground using silicon-carbide ( $\mathrm{SiC}$ ) paper. After grinding, the surface roughness $\mathrm{R}_{\mathrm{a}}$ of the 160 samples is about $30 \mathrm{~nm}$. The samples were installed on a multi-target holder to expose them simultaneously to the plasma in PSI-2. To match the PSI-2 hollow plasma profile (see [18]) and ensure the same plasma conditions on all samples, they were placed in a circle at the ame radial distance from the centre of the plasma column. The plasma flux hits the target at normal incidence and leads to heating of the samples to around $620^{\circ} \mathrm{C}$ to $650{ }^{\circ} \mathrm{C}$. The plasma is, besides the afore-mentioned residual oxygen, composed of singly ionised $\mathrm{D}+$ ions. With an ion flux of $5.5 \cdot 10^{21} \mathrm{ions} /\left(\mathrm{m}^{2} \mathrm{~s}\right)$ a total accumulated fluence of about $1 \cdot 10^{26}$ ions $/ \mathrm{m}^{2}$ in about $5 \mathrm{~h}$ was reached. More experimental details are given in [30].

Two measurements are performed to evaluate the samples erosion: mass loss $\Delta \mathrm{m}$ and surface recession $\mathrm{d}_{\mathrm{e}}$. The mass loss $\Delta \mathrm{m}$ is obtained by weighing the samples before and after plasma exposure with a Sartorius MSA225P microbalance with a resolution of $10 \mu \mathrm{g}$. The surface recession $d_{e}$ is obtained with the help of the Scanning Electron Microscope - Focussed Ion Beam (SEM-FIB) system (Carl Zeiss CrossBeam XB540): prior to plasma an orthogonal cut in the middle of a sample relative to the surface is created. For one sample an additional cut was done at the sample corner (WCrY2c). Equidistant markers are then generated on the orthogonal surface cut. The distance from the surface to the uppermost FIB marker is measured before and after plasma exposure. The distance from the receded surface to the uppermost FIB marker after plasma exposure is the surface recession $\mathrm{d}_{\mathrm{e}}$. In figure 1 the comparison of the distance between surface and uppermost FIB marker before and after plasma is shown and the surface recession $d_{e}$ is indicated.

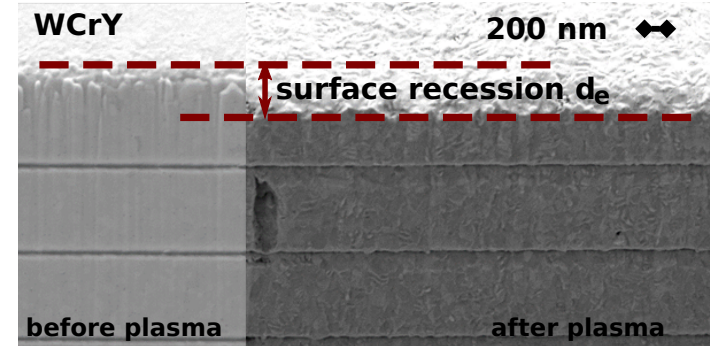

Figure 1: Comparison of the distance between surface and uppermost FIB marker before (left side) and after plasma (right side). The surface recession $d_{e}$ is the difference between these two distances.

\subsubsection{Results}

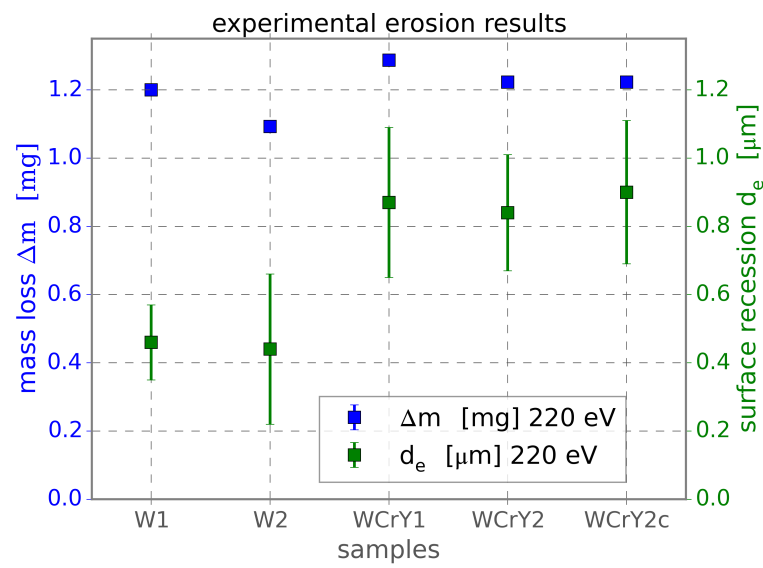

Figure 2: Comparison of mass loss $\Delta \mathrm{m}$ and surface recession $\mathrm{d}_{\mathrm{e}}$ for pure $\mathrm{W}$ and $\mathrm{WCrY}$ samples. WCrY2c is a measurement taken at the corner of WCrY2. All other measurements were done in the respective sample centre.

Results for the samples' net erosion by weight loss $\Delta \mathrm{m}$ and the surface recession $\mathrm{d}_{\mathrm{e}}$ are displayed in figure 2. Several values for $d_{e}$ are measured and the average is taken, which explains the increased measurement uncertainty. All analysis processes are explained in detail in [30]. The mass loss $\Delta \mathrm{m}$ is very similar for all samples, around $1.1 \mathrm{mg}$ for $\mathrm{W}$ and $1.2 \mathrm{mg}$ for WCrY, whereas the surface recession $d_{e}$ is nearly doubled for the WCrY with around $850 \mathrm{~nm}$ compared to the $\mathrm{W}$ samples with around $450 \mathrm{~nm}$. This result indicates faster sputtering of WCrY. The alike values for $\mathrm{d}_{\mathrm{e}}$ at the centre and corner of WCrY2 (WCrY2 and WCrY2c in figure 2, respectively) support the assumption that erosion is roughly homogeneous across the sample surface. We therefore define $\rho_{\mathrm{e}}$, the density of the sputtered material, to be the 
quotient of mass and volume loss:

$$
\begin{gathered}
\rho_{\mathrm{e}}=\Delta \mathrm{m} / \Delta \mathrm{V}=\Delta \mathrm{m} /\left(\mathrm{d}_{\mathrm{e}} \cdot \mathrm{A}\right) \\
\approx 1200 \mu \mathrm{g} /\left(850 \mathrm{~nm} \cdot 1 \mathrm{~cm}^{2}\right) \approx 14.1 \mathrm{~g} / \mathrm{cm}^{3}
\end{gathered}
$$

With an initial alloy composition of $\mathrm{W}-11.4 \mathrm{wt} \% \mathrm{Cr}-0.6 \mathrm{wt} \% \mathrm{Y}$, which is equal to 67.9 at $\% \mathrm{~W}-31.1$ at $\% \mathrm{Cr}-1$ at $\% \mathrm{Y}$, the initial theoretical density of the smart alloy is $\rho_{\mathrm{i}, 0}=15.9 \mathrm{~g} \mathrm{~cm}^{-3}$. Measurements according to the Archimedes principle confirmed an initial density of more than $98 \%$. Thus ${ }^{24}$ the minimum initial density $\rho_{i}$ of the two smart alloy samples WCrY1 and WCrY2 is $15.9 \mathrm{~g} \mathrm{~cm}^{-3} \cdot 0.98$ $=15.6 \mathrm{~g} \mathrm{~cm}^{-3}$. If the material is sputtered without significant mixing of material, the density of the eroded material $\rho_{\mathrm{e}}$ must approximately equal the initial material density $\rho_{i}$. Since the value of $\rho_{\mathrm{e}}$ is smaller than $\rho_{i}$, one can conclude that lighter elements were eroded proportionally more during plasma exposure.

\subsection{Surface composition after vacuum heating}

\subsubsection{Experiment}

The X-ray Photoelectron Spectroscopy (XPS)-device allows to heat samples to high temperatures under ultra-high-vacuum (UHV) conditions at a pressure of $1 \cdot 10^{-8}$ mbar to $1 \cdot 10^{-9}$ mbar. Moreover, the surface sensitive XPS analysis method can be used to monitor changes at the sample surface. The used XPS device is a PREVAC XPS setup equipped with an aluminium (Al) $\mathrm{K}_{\alpha}$ X-ray source at an energy of $1.4867 \mathrm{keV}$. Whereas the sputtered area amounts to $5 \mathrm{~mm} \times 5 \mathrm{~mm}$, the analysis spot measures $1.6 \mathrm{~mm} \times 5 \mathrm{~mm}$. Assuming an exponential decay of the emission intensity within the sampled material, a sampling depth of $\mathrm{z}=3 \lambda$ (the depth from which $95 \%$ of the photoemission intensity is still detected for emission normal to the target surface) is estimated to be $\mathrm{z}=5 \mathrm{~nm}$ in $\mathrm{W}$, with $\lambda$ from [17]. The XPS-setup was used to detect changes in the sample's surface composition, which are induced by heating under UHV conditions after preferential sputtering.

For this purpose one piece of sample WCrY1, ground exposure, was used. First an SEM image (figure $3 \mathrm{a}$ ) was taken, the sample was put into the XPS-device and the surface composition was measured (table 1 a). Following this, the sample was heated to $900 \mathrm{~K}$ for $1 \mathrm{~h}$,

${ }^{1}$ at\%: atomic percent, elemental fraction of total atomic composi- ${ }^{285}$ tion of alloy

3b). Then the sample was put into the XPS device again and sputter-cleaned using $5 \mathrm{keV} \mathrm{Ar}$ ions before the surface composition was again analysed (table $1 \mathrm{p}$ ). Subsequently, the sample was heated to $900 \mathrm{~K}$ for $3 \mathrm{~h}$ and another XPS analysis was carried out (table 14). Eventually, the sample was taken out and another SEM image was taken (figure 3k). One should note that the SEM pictures are not taken at exactly the same position.

\subsubsection{Results}

The elongated scratches on the untreated sample surface in figure 3 a stem from the grinding process during sample preparation. From table 19 it can be seen that there are residual impurities on the surface. The oxygen is mostly found as oxide bound to $\mathrm{W}$ and $\mathrm{Cr}$. The $5 \mathrm{keV}$ Ar-sputtering then leads to removing these impurities from the surface, the oxygen is decreased (table $1 \mathrm{p}$ ). The measured molybden (Mo) stems from the sample holder inside the XPS device. As the sample piece was with dimensions of $5 \mathrm{~mm} \times 4 \mathrm{~mm}$ only slightly larger than the measurement spot of size $1.6 \mathrm{~mm} \times 5 \mathrm{~mm}$, the analysis region can easily contain parts of the sample holder. Yet, we assume that this does not have an impact on the information we want to obtain, on the $\mathrm{W}$ and $\mathrm{Cr}$ presence in the sample surface. Further, fluorine $(\mathrm{F})$ was found on the surface. This element has also been found by other analysis methods for samples produced and prepared around the same time. We assume that this fluorine stems from residual impurities on the equipment used for ultrasonic cleaning after the sample preparation procedure. The Ar-sputtering leads not only to a removal of an approximately $3 \mathrm{~nm}$ thick surface layer, but also to a characteristic surface morphology of small holes and elongated craters along the grain boundaries. The microstructure of WCrY features yttrium and oxide containing particles located at grain boundaries [30]. These are known to have the size of the order of tens of $\mathrm{nm}$. The small black holes as well as the longer elongated craters are characteristic for smart alloys after being exposed to (plasma) ion irradiation and have been investigated in [30] and [29]. These features are caused by preferential sputtering of lighter elements and can still be seen on the SEM picture in figure 3 . taken after heating of the sample for $3 \mathrm{~h}$. It can be excluded that these features stem from heating the sample to $900 \mathrm{~K}$ since they are not observed in figure $3 \mathrm{p}$ after heating to this temperature for $1 \mathrm{~h}$. For the XPSanalysis the $\mathrm{Y}$ content of the sample $(1.0 \mathrm{at} \%)$ is close to the detection limit and not detectable after the Arsputtering (table 1 $\mathrm{b}$ ) due to preferential sputtering. The XPS-analysis results after heating for $3 \mathrm{~h}$ are shown in 


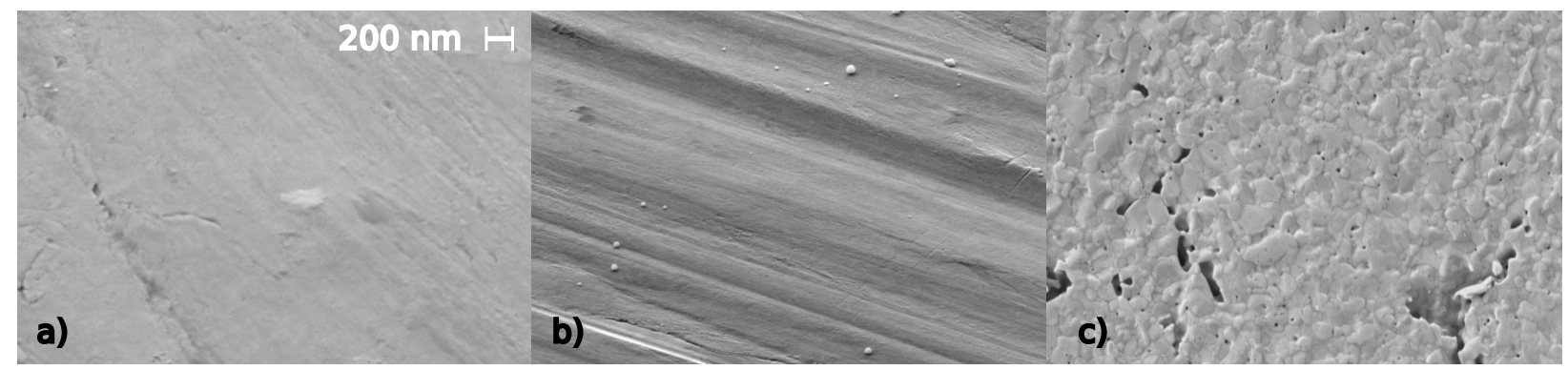

Figure 3: SEM images of the surface of the WCrY sample a) untreated, b) after heating to $900 \mathrm{~K}$ for $1 \mathrm{~h}$ and c) after Ar-sputtering and subsequent heating to $900 \mathrm{~K}$ for $3 \mathrm{~h}$ in UHV conditions.

\begin{tabular}{ccccc} 
& W [at\%] & Cr [at\%] & Y [at\%] & other elements [at\%] \\
\hline a) $12.4 \pm 0.2$ & $3.4 \pm 0.2$ & $0.9 \pm 0.1$ & $34.0 \pm 0.5(\mathrm{O}), 26.2 \pm 0.8(\mathrm{C})$ \\
& & & $0.4 \pm 0.0(\mathrm{Mo}), 23.0 \pm 0.5(\mathrm{~F})$ \\
b) $73.0 \pm 1.1$ & $14.9 \pm 0.7$ & 0.0 & $10.7 \pm 1.2(\mathrm{O}), 1.4 \pm 0.2(\mathrm{Mo})$ \\
& & & \\
c) $63.8 \pm 0.8$ & $17.5 \pm 0.6$ & 0.0 & $18.1 \pm 0.9(\mathrm{O}), 0.7 \pm 0.2(\mathrm{Mo})$
\end{tabular}

Table 1: XPS-analysis of a piece of WCrY1 a) before Ar-sputtering, b) after Ar-sputtering and c) after Ar-sputtering and heating for $3 \mathrm{~h}$ to $627^{\circ} \mathrm{C}$

table 17. The $\mathrm{W}$ surface coverage decreased and the oxygen as well as slightly the $\mathrm{Cr}$ surface coverage are increased after heating. Again, $\mathrm{W}$ and $\mathrm{Cr}$ are present in partly oxidised state. Hence, in UHV conditions and when the sample is heated after preferential sputtering $\mathrm{Cr}$ diffuses to the surface to form oxides so that less $\mathrm{W}$ is present.

\section{SDTrimSP Modelling}

\subsection{Model description}

The Monte-Carlo code SDTrimSP [24] makes use of the Binary-Collision-Approximation (BCA) to simulate ion irradiation of amorphous targets. The underlying physics is described in [6]. In the dynamic version a one-dimensional target made up of dynamically thickening or shrinking layers is employed. The ion projectiles successively shot into the target create recoils which in turn lead to reordering of atoms. Eventually, sputtering of atoms in the surface layer is caused if the energy transferred to these atoms is greater than the surface binding energy. Concentration gradients are induced in case of preferential sputtering for mixed materials. This means that the sputter yield, the number of atoms sputtered per incoming projectile, for one atomic species is higher than the yield of the other atomic species of the mixed material. With diffusion added to the model, the depleted element is transported towards the surface to counteract the build-up of these gradients. Owing to the usage of the dynamic version, we are able to look into the evolution of the alloy's near-surface composition. Since a one-dimensional target model is used, we do not reproduce the specific microstructure of the WCrY material but rather assume an amorphous target structure with a composition of 67.9 at $\% \mathrm{~W}$ 31.1 at $\% \mathrm{Cr}-1$ at $\%$ Y. The target is initially subdivided into equidistant $2.5 \AA$ thick layers of the same composition. In a calculation step, a certain number of projectile ions, i.e. a certain fluence, is shot onto the target and their impact is calculated. After each such fluence step the target composition is updated according to the change in the number of the respective atomic species in each layer. If the composition is changed due to implantation or reordering of the recoils, this, in turn, causes a change in the atomic density. Implantation and recoils further result in an increase or ,in case of sputtering, decrease of the layer thickness. Summing over the changes in thickness of all layers leads to growth or shrinkage of the target. If erosion dominates the summation results in shrinkage, i.e. the surface recesses. For the calculations normal incidence of the bombarding ions is assumed. The samples' surface roughness 
of about $30 \mathrm{~nm}$ cannot be reproduced with the one- 305 dimensional model. This should not have a large impact on the erosion results for the case of normal incidence irradiation.

In SDTrimSP [24] thermal diffusion between adjacent target layers can be included into the model. For ${ }_{310}$ this purpose the temperature-dependent diffusion coefficients must be specified. Generally, diffusion is induced by concentration gradients of an element within a material. This is expressed by equation (1), which depicts the evolution of the element(i)-specific concen- 315 tration $\mathrm{c}$ at layer $\mathrm{x}$. The diffusion coefficient in equation (2) increases exponentially with temperature T. Diffusive transport across the surface boundary is set to zero. However, if atoms of the first layers get sputtered, the induced concentration gradient initiates diffusion from subsurface layers. The physical sputtering process within the model depends solely on the number of ions arriving at the target and not on the time as collisions are treated successively. In contrast, diffusion is a time-dependent process. Therefore, the ion flux, 325 i.e. the number of ions arriving at the target per second, enforces a time dependence. The time for a fluence step results from equation (3), this time is then used to calculate a diffusion step. Calculated recoils result in changes of the concentrations $\mathrm{c}$ of the elements $\mathrm{i}$ within each layer $\mathrm{x}$. Based on this concentration change, the overall layer-specific diffusion coefficient $\eta(x, i)$ (see equation (4)) is calculated. This is done by summing over the concentrations and diffusion coefficients of element $\mathrm{i}$ within material $\mathrm{j}$ for all elements $\mathrm{j}$.

$$
\begin{gathered}
\frac{\partial c(i)}{\partial t}=-\frac{\partial\left(\eta(x, i) \cdot \frac{\partial c(x, i)}{\partial x}\right)}{\partial x} \\
D(i, j)=D_{0}(i, j) \cdot \exp \left(-\frac{Q_{a}(i, j)}{k_{B} T}\right) \\
\Delta t=\frac{\Delta \text { fluence }\left[m^{-2}\right]}{\text { flux }\left[m^{-2} s^{-1}\right]} \\
\eta(x, i)=\sum c(x, j) \cdot D(i, j)
\end{gathered}
$$

According to equation 2 two material-specific parameters have to be specified to assign a diffusion coefficient $D(i, j)$ for element $i$ in element $j$ : the activation energy $Q_{a}$ and the diffusion constant $D_{0}(i, j)$. Both are not known for the alloy WCrY. In order to vary only $\mathrm{D}_{0}(\mathrm{i}, \mathrm{j})$ for the simulations, we do not specify $\mathrm{Q}_{\mathrm{a}}$ (see [23] for detailed explanation). Therefore, the simulations are only valid for the sample temperature present in the experiment. In that way a parameter scan for $D_{0}(i, j)$ can be performed. For the simple physical sputtering process, temperature is not taken into account within the BCA. The implementation of the algorithm solving the diffusion equation and further details are described in [35].

Lighter alloyed elements are preferentially sputtered, which leads to a continuous change of the surface stoichiometry during ion irradiation until a steady state is reached [32]. For this state the ratio of the sputter yields is stoichiometric [1]. This means for dynamical SDTrimSP runs partial sputter yields converge with increasing fluence (see figure $4 \mathrm{~b}$ ). In order to save computational time, simulations were carried out to a certain fluence and then extrapolated to the experimental fluence. Simulations shown here calculate $10 \%$ of the experimental fluence, which is $1 \cdot 10^{26}$ ions $/ \mathrm{m}^{2} \times$ $0.1=1 \cdot 10^{25}$ ions $/ \mathrm{m}^{2}$, assuming steady state has approximately been reached at this point. The last 10 values of this simulation are then used to linearly extrapolate the surface recession: the slope of a line intersecting these last 10 values is assumed to be constant until $100 \%$ of the experimental fluence.

The mass loss is obtained from the simulation output by integration of the partial yield curves. Mass loss values were extrapolated using the partial sputter yields of $\mathrm{W}$, $\mathrm{Cr}$ and $\mathrm{Y}$ at the last fluence step.

Experimental values are used for the adaptation of $\mathrm{D}_{0}(\mathrm{i}, \mathrm{j})$ : the experimentally obtained surface recession $\mathrm{d}_{\mathrm{e}}$ (see figure 2) is taken to be $450 \mathrm{~nm}$ for pure $\mathrm{W}$ and $850 \mathrm{~nm}$ for WCrY. Mass loss $\Delta \mathrm{m}$ reference values for $\mathrm{W}$

335 and $\mathrm{WCrY}$ are $1.1 \mathrm{mg}$ and $1.2 \mathrm{mg}$, respectively. Both the measured mass loss and even more the surface recession are subject to measurement uncertainties (see figure 2), which is considered for determining the model parameters. Neither preferential sputtering nor diffusion have to be considered for the erosion yield of pure W samples.

\subsection{Model results}

In the following the effect of $\mathrm{D}$ ion irradiation, oxygen plasma content and $\mathrm{Cr}$ diffusion is presented according to their order of implementation in the model. Subsequently, effects of D retention, surface binding energies and possible other factors are examined.

\subsubsection{Pure D irradiation}

Sputter thresholds for pure $\mathrm{W}, \mathrm{Cr}$ and Y calculated with SDTrimSP in static mode are shown in table 2 . The thresholds for $\mathrm{W}$ and $\mathrm{Cr}$ are about $220 \mathrm{eV}$ and $35 \mathrm{eV}$, respectively, and are approximately in agreement with the threshold values as presented in [33], 216 eV for $\mathrm{W}$ and 


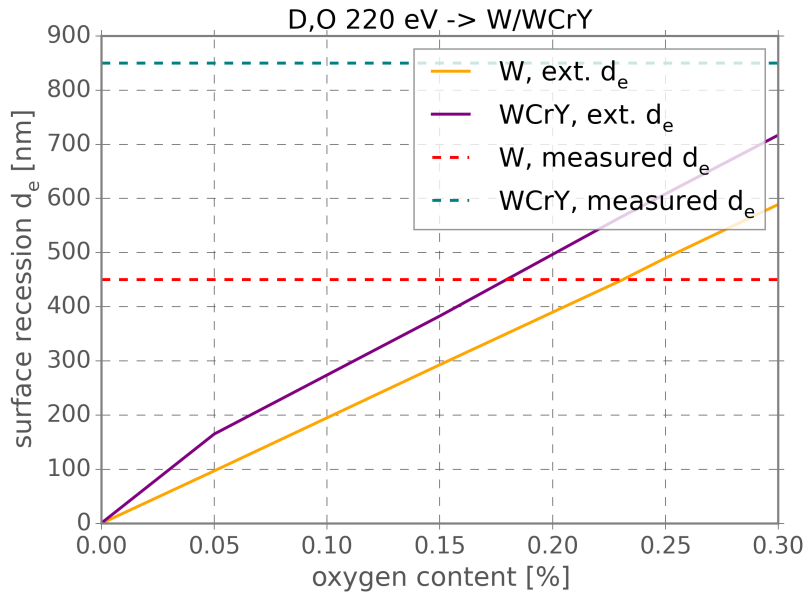

(a)

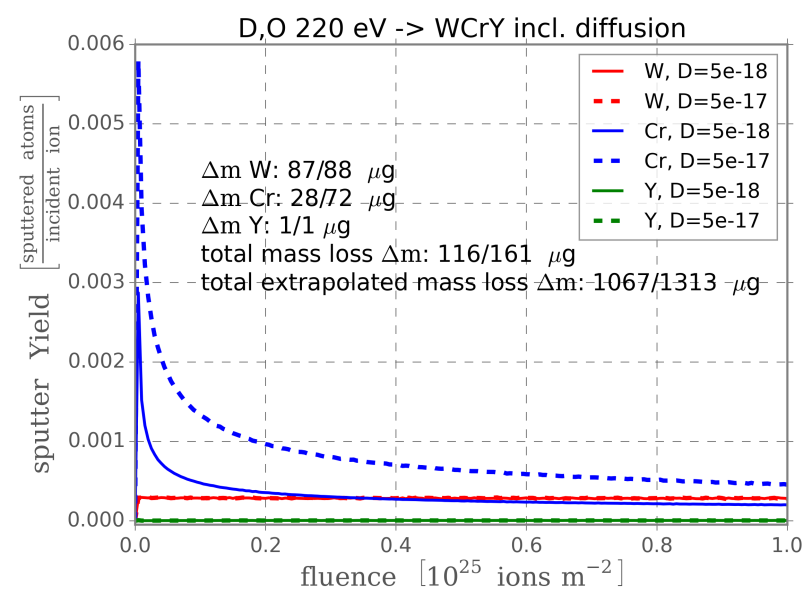

(b)

Figure 4: SDTrimSP calculated surface recessions [nm] under D, O $220 \mathrm{eV}$ ion bombardment: a) values for pure W and WCrY with mixed $\mathrm{D}, \mathrm{O}$ ion irradiation with variation of the oxygen content $O$ extrapolated (ext.) to the experimental fluence of $1 \cdot 10^{26} \mathrm{ions} / \mathrm{m}^{2}$. Experimentally measured values for the surface recession $\mathrm{d}_{\mathrm{e}}$ of $\mathrm{W}$ and $\mathrm{WCrY}$ are indicated by horizontal dotted lines. b) simulated partial sputter yields of $\mathrm{W}, \mathrm{Cr}$ and $\mathrm{Y}$ for WCrY with the diffusion coefficient of Cr in WCrY, $\mathrm{D}_{\mathrm{Cr}, \mathrm{WCrY}}$, set to $5 \cdot 10^{-18} \mathrm{~m}^{2} / \mathrm{s}$ (continuous lines) and set to $5 \cdot 10^{-17} \mathrm{~m}^{2} / \mathrm{s}$ (dashed lines). The mass loss per element is obtained via integration of the partial yield curves. Here the first value is the mass loss for $\mathrm{D}_{\mathrm{Cr} \text {, } \mathrm{WCrY}} \operatorname{set}$ to $5 \cdot 10^{-18} \mathrm{~m}^{2} / \mathrm{s}$ and the second one the mass loss for $\mathrm{D}_{\mathrm{Cr}, \mathrm{WCrY}}$ set to $5 \cdot 10^{-17} \mathrm{~m}^{2} / \mathrm{s}$.

\begin{tabular}{c|ccc} 
target & \multicolumn{3}{|c}{ bombarding ion species } \\
& $\mathrm{D}$ & $\mathrm{O}$ & $\mathrm{Ar}$ \\
\hline $\mathrm{W}$ & 220 & 45 & 35 \\
$\mathrm{Cr}$ & 35 & 20 & 20 \\
$\mathrm{Y}$ & 60 & 15 & 20
\end{tabular}

Table 2: Thresholds [eV] for pure elements W, Cr and Y sputtered by $\mathrm{D}, \mathrm{O}$ and $\mathrm{Ar}$ as calculated with SDTrimSP at normal incidence using surface binding energies of $8.79 \mathrm{eV}, 4.10 \mathrm{eV}$ and $4.36 \mathrm{eV}$ for $\mathrm{W}, \mathrm{Cr}$ and $\mathrm{Y}$, respectively.

$34 \mathrm{eV}$ for $\mathrm{Cr}$. Oxygen sputter thresholds are considerably lower according to SDTrimSP: $45 \mathrm{eV}$ for $\mathrm{W}$ and $20 \mathrm{eV}$ for $\mathrm{Cr}$. Especially at D ion energies close to or below the threshold, the effect of impurity oxygen becomes important [13]. Consequently, it can be expected that already small amounts of $\mathrm{O}$ in the target ion flux have a large impact on the material's erosion. Additionally, in the vicinity of $\mathrm{Cr}$ atoms, $\mathrm{W}$ atoms can get sputtered more easily due to an intermediate $\mathrm{Cr}-\mathrm{W}$ collision [30]: according to the BCA the maximum elastic energy transfer between two colliding atoms 1 and 2 is

$$
\begin{gathered}
\gamma=4 \mathrm{~m}_{1} \mathrm{~m}_{2} /\left(\mathrm{m}_{1}+\mathrm{m}_{2}\right)^{2} \\
\gamma_{\mathrm{D}, \mathrm{W}} \approx 0.04, \gamma_{\mathrm{D}, \mathrm{Cr}} \approx 0.14, \gamma_{\mathrm{Cr}, \mathrm{W}} \approx 0.69 \\
\rightarrow \gamma_{\mathrm{D}, \mathrm{Cr}, \mathrm{W}} \approx 0.10
\end{gathered}
$$

With an intermediate Cr-W collision instead of the di350 tor $\gamma$ is increased from 0.04 to 0.10 . Thus in the vicinity of $\mathrm{Cr}$ atoms $\mathrm{W}$ sputtering is augmented. This effect is automatically included in the SDTrimSP model.

Surface recession results for W (yellow line) and WCrY (purple line) in pure D are shown in figure 4a, the first data point at $\mathrm{x}=0$ corresponds to an oxygen amount of $0.0 \%$ in the plasma. For $\mathrm{W}$ the simulated surface recession amounts to only below $1 \mathrm{~nm}$. This is far below the experimental value of $450 \mathrm{~nm}$ (red dotted line). Consequently, other factors influencing the sputter yield have to be considered.

\subsubsection{Mixed D, O irradiation}

The plasma oxygen content was varied in a next step. This value likely amounts to a few tenths of one percent of the plasma ion flux in PSI-2 (see chapter2). In figure 4a surface recession versus varying oxygen content is displayed for both pure W and WCrY. Experimental surface recession of pure $\mathrm{W}(450 \mathrm{~nm})$ is reproduced assuming a plasma composition of $99.73 \% \mathrm{D}$ and $0.23 \% \mathrm{O}$, here the red dotted and yellow line intersect. Hence, this composition was used for further calculations. Whereas the experimentally obtained surface recession of pure $\mathrm{W}$ is matched by including oxygen, the WCrY surface recession of about $565 \mathrm{~nm}$ is far below the experimental 
(WCrY, measured $\mathrm{d}_{\mathrm{e}}$ ) and the purple line (WCrY, ext. $\mathrm{d}_{\mathrm{e}}$ ) in figure $4 \mathrm{a}$

\subsubsection{Mixed $D, O$ irradiation including diffusion}

To increase the sputtering of lighter alloying elements during the ion bombardment, these must be transported to the surface. The transport process was simulated by including thermal diffusion. For the variation of the diffusion coefficient of $\mathrm{Cr}$ in $\mathrm{WCrY}, \mathrm{D}_{\mathrm{Cr}} \mathrm{WCrY}$, the oxygen ${ }_{435}$ content of the plasma is fixed to $0.23 \%$.

In order to calculate, besides the surface recession, also the mass loss from the simulations, the partial sputter yields are used. The evolution of the partial sputter yields of $\mathrm{W}, \mathrm{Cr}$ and $\mathrm{Y}$ is displayed in figure $4 \mathrm{~b}$ up to a fluence of $1 \cdot 10^{25}$ ions $/ \mathrm{m}^{2}$ for the diffusion coefficient $\mathrm{D}_{\mathrm{Cr} \text { WCrY }}$ set to $5 \cdot 10^{-18} \mathrm{~m}^{2} / \mathrm{s}$ (continuous lines) and set to $5 \cdot 10^{-17} \mathrm{~m}^{2} / \mathrm{s}$ (dashed lines). All yield curves show an asymptotic trend towards increasing fluence, i.e. the sputter yields converge. The total mass loss is the sum of the partial mass losses for $\mathrm{W}, \mathrm{Cr}$ and $\mathrm{Y}$. Up to the calculated fluence the mass loss is obtained via integration of the yield curves, the yield obtained for the last fluence step is used for extrapolating the mass loss up to higher fluencies. On average, most of the mass loss 450 is caused by W sputtering, whereas $\mathrm{Cr}$ sputtering is pre400 dominant at the beginning of the irradiation. The $\mathrm{Cr}$ partial sputtering yield is increased while $\mathrm{W}$ sputtering is at a low level because at first more $\mathrm{Cr}$ is present at the surface. In this state surface recession is acceler- ${ }_{455}$ ated compared to that of pure W. In the course of the rradiation $\mathrm{Cr}$ gets preferentially sputtered and less $\mathrm{Cr}$ atoms are present at the surface so that then more $\mathrm{W}$ is sputtered. Adding diffusion to the model leads to $\mathrm{Cr}$ transport towards the surface in case preferential sput- 460 tering creates $\mathrm{Cr}$ concentration gradients. For a higher ension coefficient the $\mathrm{Cr}$ transport towards the surface is stronger, provided a $\mathrm{Cr}$ concentration gradient exists. Stronger diffusion results in less pronounced $\mathrm{Cr}$ depletion at the alloy's surface, which means that the sputter ${ }_{465}$ yield of $\mathrm{Cr}$ is higher than that of $\mathrm{W}$ up to a higher flu415 ence. Thus the overall mass loss is increased $(1313 \mu \mathrm{g}$ at $\mathrm{D}_{\mathrm{Cr}, \mathrm{WCrY}}=5 \cdot 10^{-17} \mathrm{~m}^{2} / \mathrm{s}$ compared to $1067 \mu \mathrm{g}$ at $\mathrm{D}_{\mathrm{Cr}, \mathrm{WCrY}}=5 \cdot 10^{-18} \mathrm{~m}^{2} / \mathrm{s}$ ) as the mass loss caused by $\mathrm{Cr}$ is increased ( $72 \mu \mathrm{g}$ compared to $28 \mu \mathrm{g}$ ). Stronger diffusion can be caused either by a higher diffusion coef- 470 ficient or by higher sample temperatures. However, the temperature was not varied in the simulations. Due to the small Y content in the sample, mass loss caused by Y sputtering does not play a significant role.

The diffusion coefficient was varied over several orders 475 of magnitude. Surface recession values are calculated up to a fluence of $1 \cdot 10^{25} \mathrm{ions} / \mathrm{m}^{2}$ and then extrapo- lated, results are shown in figure 5a At $10 \%$ of the experimental surface recession, i.e. $85 \mathrm{~nm}$, the simulation results for $10 \%$ of the experimental fluence match 430 when choosing $\mathrm{D}_{\mathrm{Cr}, \mathrm{WCrY}}=5 \cdot 10^{-18} \mathrm{~m}^{2} / \mathrm{s}$. However, extrapolating the results of this simulation run to $100 \%$ of the experimental fluence yields a surface recession value below the experimental one as can be seen in $5 b$ the extrapolated simulation yields a value of $723 \mathrm{~nm}$ (green line), which is below the experimental value of $850 \mathrm{~nm}$ (teal dotted line). With the aim of comparing extrapolated values to non-extrapolated ones, one calculation was performed for $\mathrm{W}$ (yellow line) and one for WCrY (purple line) up to a fluence of $1 \cdot 10^{26}$ ions $/ \mathrm{m}^{2}$. The diffusion coefficient $\mathrm{D}_{\mathrm{Cr}, \mathrm{WCrY}}$ was set to $5 \cdot 10^{-18} \mathrm{~m}^{2} / \mathrm{s}$. Results are also shown in figure $5 \mathrm{~b}$ : the non-extrapolated simulation yields a value of $660 \mathrm{~nm}$ (purple line), which is below the extrapolated value of $723 \mathrm{~nm}$ (green line). This is because the yield curves have not fully reached stationary conditions at the end of the simulated fluence. Since the surface recession is not yet strictly linear for WCrY, the model results deviate for later steps. Thus for more exactly determining the numerical value of the diffusion coefficient, simulations should be extended to $100 \%$ of the experimental fluence. Yet, this would acquire very long computation times. Moreover, one has to take into account that the experimental surface and mass loss values are subject to measurement uncertainties which have an impact on the value of the diffusion coefficient as well. Consequently, for assigning a conclusive value to the diffusion coefficient, both the surface recession and the mass loss were considered.

The blue shaded bar in figure $5 \mathrm{a}$ indicates the range for the diffusion coefficient in which extrapolated simulation results either match the experimentally found surface recession $d_{e}$ (left edge of the blue shaded bar) or the experimentally found mass loss $\Delta \mathrm{m}$ (right edge of the blue shaded bar). The diffusion coefficient has to be set to a value within the blue-shaded range in figure $5 \mathrm{a}$, 2 to $3 \cdot 10^{-17} \mathrm{~m}^{2} / \mathrm{s}$

\subsection{Role of surface binding energy and retention}

A further factor influencing the erosion yield of a material is the surface binding energy $\mathrm{E}_{\mathrm{SB}}$. This is the energy that a target surface atom needs to overcome to get sputtered. Generally, it is known that alloying leads to a decrease in surface binding energy. However, $\mathrm{E}_{\mathrm{SB}}$ values for WCrY are not known. Commonly, for BCA models the heat of sublimation energy is used as an approximation for $\mathrm{E}_{\mathrm{SB}}$, as the actual $\mathrm{E}_{\mathrm{SB}}$ values are unknown [39]. For $\mathrm{W}$ the default value in SDTrimSP tables is $8.79 \mathrm{eV}$. Lowering of $\mathrm{E}_{\mathrm{SB}}$ to e.g. $6 \mathrm{eV}$ for $\mathrm{W}$ in 


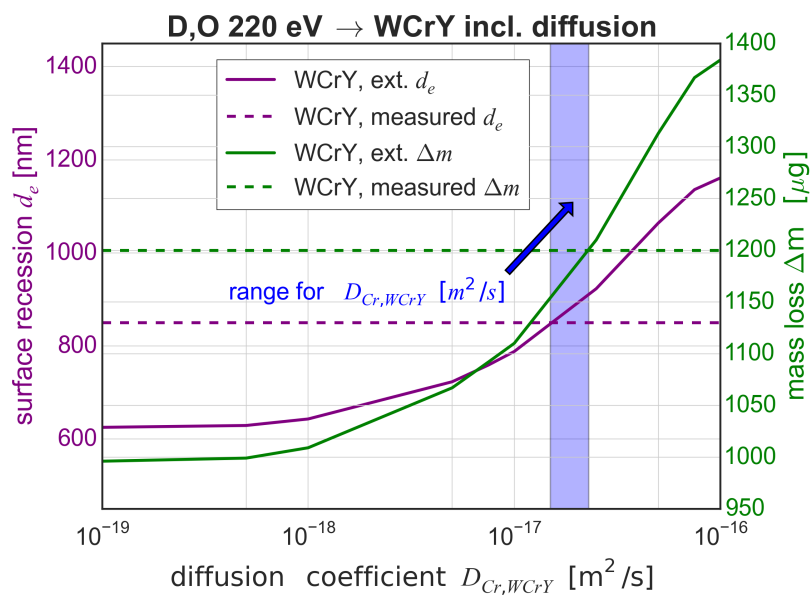

(a)

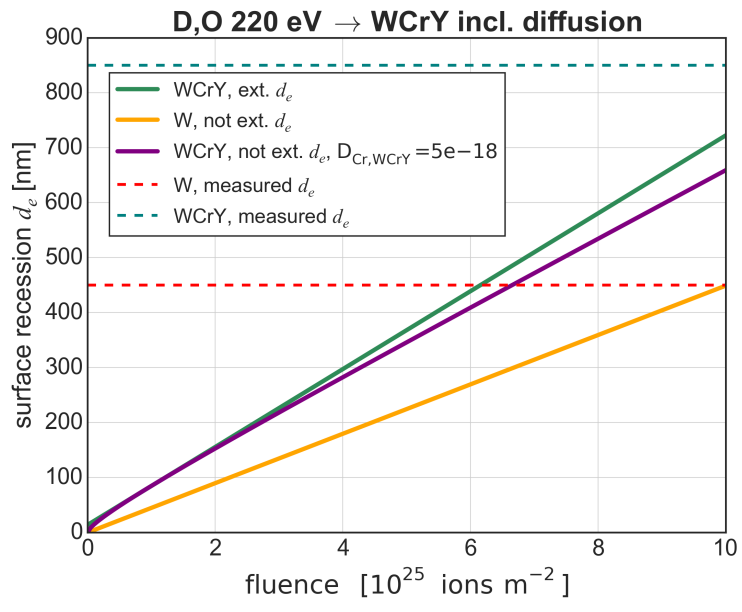

(b)

Figure 5: SDTrimSP calculations under D, O $(0.23 \% \mathrm{O}) 220 \mathrm{eV}$ ion bombardment. a) shows the extrapolated (ext.) surface recession (sr) and mass loss (ml) for WCrY with varying diffusion coefficient. Experimentally measured values for the surface recession $\mathrm{d}_{\mathrm{e}}$ and the mass loss $\Delta \mathrm{m}$ of WCrY are indicated by horizontal dotted lines. If the diffusion coefficient is set to a value within the blue shaded range, simulation and experimental results match. b) surface recession of pure $\mathrm{W}$ and WCrY (including diffusion with $\mathrm{D}_{\mathrm{Cr} \text { WCrY }}=5 \cdot 10^{-18} \mathrm{~m}^{2} / \mathrm{s}$ ) calculated for the experimental fluence of $1 \cdot 10^{26}$ ions $/ \mathrm{m}^{2}$ and not extrapolated (W and WCrY not ext.) and calculated for a fluence of $1 \cdot 10^{25}$ ions $/ \mathrm{m}^{2}$ and then extrapolated to the experimental fluence (WCrY ext.). Experimentally measured values for the surface recession $\mathrm{d}_{\mathrm{e}}$ of $\mathrm{W}$ and $\mathrm{WCrY}$ are indicated by horizontal dotted lines.

WCrY at a plasma oxygen content of $0.23 \%$ leads to an 505 extrapolated surface recession of about $3 \mu \mathrm{m}$, at a mass loss of about $4.7 \mathrm{mg}$. This would mean a density of the eroded material of $\rho_{\mathrm{e}}=15.7 \mathrm{~g} \mathrm{~cm}^{-3}$, which is about the initial density of the alloy (see chapter 2.1.2. Hence, a much lower surface binding energy of $\mathrm{W}$ in a mixed ma- 510 terial may contribute to enhanced overall erosion. Yet, explaining the reduced density of the eroded material, Cr-transport to the surface is needed.

For plasma-facing materials considering $\mathrm{D}$ retention is important. At the high sample temperatures of $620^{\circ} \mathrm{C}$ to $650^{\circ} \mathrm{C}$ during the experiment, $\mathrm{D}$ is easily released again and its content is hence very low when measured after- 51 wards. [28] states that a higher intrinsic trap density in W-tantalum(Ta) alloys raises retention compared to pure $\mathrm{W}$, while [40] finds no difference in the amount of retained $\mathrm{H}$ between $\mathrm{W}$ and $\mathrm{W}-\mathrm{Ta}$. D retention was found to be just slightly increased for WCrY compared to W when measured after the exposure [30]. Still, for a moment it may be present in the target during the exposure and hence influence the sputtering behaviour. When including D retention into the SDTrimSP model the target density is reduced with increasing the number of re- 525 tained light $\mathrm{D}$ atoms. In that case less $\mathrm{W}$ is located at the target surface leading to a reduced W sputter yield. However, since there is no measurement of $\mathrm{D}$ retention during plasma exposure, the model is just used to qual- itatively assess the influence of $\mathrm{D}$ retention on erosion yields.

In [11] and [26] a reduced energy threshold for oxidised $\mathrm{W}$ sputtered by light ions as compared to pure $\mathrm{W}$ is reported. This results from a decrease in the binding energy of tungsten oxide molecules. In SDTrimSP only binary atom collisions are considered and hence the effect of possible oxide formation is not taken into account.

\section{Discussion}

Comparison of experimental and simulation results for D ion irradiation on W and WCrY, including the effect of residual oxygen in the plasma, highlights the need for diffusion in the current model. By using the fact that, unlike a WCrY sample, the composition of a pure $\mathrm{W}$ sample does not change during plasma exposure, a value for the oxygen content of the plasma can be set (chapter 3.2.2). Here re-deposition and selfsputtering are not taken into account since results of the experimental erosion measurements represent the net erosion, not the gross erosion. SDTrimSP simulations do not include the effect of re-deposition. For estimating the effect of self-sputtering and re-deposition of $\mathrm{W}$ and $\mathrm{Cr}$ in PSI-2, ERO modelling was performed in other studies. ERO is a 3D Monte Carlo code used for simulating transport of sputtered particles. As described in 
[8] there exists a version adapted to the PSI-2 geometry. Calculations employing this version and plasma parameters taken from measurements during the experiment ${ }_{585}$ were executed for pure $\mathrm{W}$ and $\mathrm{Cr}$ targets. The fraction of re-deposited material was calculated to be around $26 \%$ in the case of $\mathrm{W}$ and $14 \%$ for pure Cr. Hence an error is introduced as SDTrimSP calculates the gross erosion. Consequently, more $\mathrm{W}$ as well as WCrY may have been sputtered during the plasma exposure and the plasma oxygen content may be higher than $0.23 \%$.

After heating a $\mathrm{WCrY}$ sample for $3 \mathrm{~h}$ to $627^{\circ} \mathrm{C}$ in the UHV conditions of the XPS device no $\mathrm{W}$-oxides were found on the surface 2.2.2. Yet an increased $\mathrm{Cr}$ and O surface content was detected (see table 1 in 2.2.2). oxides already at very low vacuum pressures. Potentially also during plasma exposure Cr-oxides may have been formed, adding to the effect of preferential sputtering of Cr. In SDTrimSP only binary atom collisions are considered, possible oxide formation is not taken into account.

In [35] simulations of coupled sputter-diffusion effects for a W-Fe system are presented. It was found out that when choosing a too narrow slab width in the target mede algorithm used to solve the diffusion equa- 605 tions is prone to oscillatory behaviour. This is then reflected by oscillations in the surface concentrations. For the simulations presented in this work oscillatory behaviour was found neither for sputter yields nor for sur-

560 face concentrations. This is why the initial layer width 610 of $2.5 \AA$ used in the target model is assumed to be appropriate to prevent the addition of synthetic effects to the Cr-diffusion process. The sharp decrease of the $\mathrm{Cr}$ partial sputter yield (figure 4b) can be ascribed to a Wenriched layer at the target surface. Similar experimen- 615 tal findings are presented for $\mathrm{W}$-enrichment in CLF1 steel in [25] or EUROFER in [32].

Looking more closely on the numerical value for the diffusion coefficient, the following considerations were taken (see also 3.2.3): the partial sputter yields are ap- 620 proximately constant at $10 \%$ of the experimental fluence and were thus used for extrapolation to $100 \%$ of the experimental fluence to reduce computational time. Since the partial yields have not entirely converged, especially for $\mathrm{Cr}$, values extrapolated to $100 \%$ remain 625 below the reference values for WCrY. The surface recession is approximately linear only when $\mathrm{W}$ partial sputtering dominates, after the lighter alloying elements have been depleted. Extending all simulations to $100 \%$ experimental fluence would yield more exact surface re- 630 cession and mass loss values at much higher computational costs. However, experimental measurement un- certainties and neglecting the influence of further factors as laid out in chapter 3.3 , counteract the benefit of extending all simulations. Neglecting the influence of D retention, the $\mathrm{Cr}$-diffusion in $\mathrm{WCrY}, \mathrm{D}_{\mathrm{Cr}} \mathrm{WCrY}$, is likely greater than $5 \cdot 10^{-18} \mathrm{~m}^{2} / \mathrm{s}$ and situated within the range of 2 to $3 \cdot 10^{-17} \mathrm{~m}^{2} / \mathrm{s}$.

\section{Summary and outlook}

WCrY and W samples were simultaneously exposed to pure D plasma at an ion energy of $220 \mathrm{eV}$ in the linear plasma device PSI-2, net erosion and surface recession were measured. Already from calculating the density of the eroded material $\rho_{\mathrm{e}}$ it becomes evident that in case of WCrY lighter elements must have been transported towards the surface to be continuously sputtered and to lower the density of the eroded material (see 2.1.2). The XPS-treatment in chapter 2.2.2 gives experimental evidence for $\mathrm{Cr}$ diffusion to the surface at elevated sample temperatures after preferential sputtering. Diffusion of $\mathrm{Cr}$ in WCrY during ion irradiation causes increased surface recession and mass loss. Onedimensional SDTrimSP simulations showed that the effect of Cr-diffusion contributes to the erosion of WCrY samples during the plasma exposure with sample temperatures of $620^{\circ} \mathrm{C}$ to $650{ }^{\circ} \mathrm{C}$. Comparison of experimental and model results, taking into account also the plasma oxygen content, yields a diffusion coefficient of $\mathrm{Cr}$ in WCrY of the order of $\mathrm{D}_{\mathrm{Cr}, \mathrm{WCrY}}=1 \cdot 10^{-17} \mathrm{~m}^{2} / \mathrm{s}$. This value is valid for the temperature of the conducted plasma experiment.

The temperature of the first wall of future fusion devices such as DEMO could differ from the estimated temperatures of around $600{ }^{\circ} \mathrm{C}$ to $700{ }^{\circ} \mathrm{C}$ or fluctuate. Therefore, it is important to consider the influence of different temperature ranges on possible processes within the wall material. Temperatures of about $1000^{\circ} \mathrm{C}$ will lead to both $\mathrm{W}$ - and Cr-oxidation. The latter subsequently leads to enhanced $\mathrm{Cr}$ erosion due to the elevated sputter yields of the oxide. According to [37] the damage of PFCs due to increased heat loads during disruptions as compared to regular operation is of great concern for DEMO. A higher temperature can lead to enhanced $\mathrm{Cr}$ transport towards the surface, which results in increased erosion yields. Similarly, the effect of higher temperatures for the iron-tungsten (FeW) system is explained in [35]: high-Cr mobility suppresses build-up of gradients and therefore $\mathrm{W}$ enrichment. To investigate the temperature dependence of the W surface enrichment in WCrY, plasma exposure of samples at much higher and much lower temperatures than in the here described experiment in PSI-2 is envisaged. Better knowledge of the ac- 
tivation energy $\mathrm{Q}_{\mathrm{a}}$ for Cr-diffusion in the WCrY-system would help investigating the temperature dependence, 685 yet this requires additional designated experiments and models.

For a material to be used as DEMO first wall material additional effects have to be considered: Effects of neutron irradiation induced transmutation of $\mathrm{W}$ and compositional changes on the material properties of $\mathrm{W}$ are complex and need to be considered for fusion operation [12]. For $\mathrm{W}$ alloys impacting neutrons can affect the different alloying elements in diverse ways. A good understanding of irradiation-induced segregation to grain boundaries of alloying and impurity elements is important also regarding mechanical properties of the material [9]. Especially Cr-segregation to the surface, contributing to the thermally activated diffusion, could, besides mechanical properties, affect the sputtering and oxidation performance of smart alloys.

The fraction of loss power radiated from the main chamber, the SOL and the divertor region has to be increased to solve the divertor power exhaust problem in DEMO. For this purpose impurity seeding is necessary [38]. Connected to the usage of different impurity species at different locations in the DEMO reactor, the gross and 710 net erosion of PFCs at different locations has to be assessed. Here again for the smart alloys higher sputter yields of e.g. Ar (see table 2), $\mathrm{Kr}$ and $\mathrm{Xe}$ as compared to $\mathrm{D}$ and $\mathrm{T}$ may prevent the build-up of a sufficiently thick $\mathrm{W}$-enriched layer. Enhanced $\mathrm{Cr}$ sputtering at temperatures of above $600{ }^{\circ} \mathrm{C}$ may lead to a significantly increased erosion of smart alloys as first wall components in comparison to a pure $\mathrm{W}$ first wall. Yet, impurity sputtering as well as CX neutrals and self-sputtering are a threat not only to WCrY but also to pure $\mathrm{W}$ wall components.

\section{References}

[1] R. Behrisch and W. Eckstein, eds. Sputtering by Particle Bombardment: Experiments and Computer Calculations from Threshold to MeV Energies. Vol. 110. Topics in Applied Physics. Berlin Heidelberg: Springer-Verlag GmbH, 2007. 730 ISBN: 978-3-540-44502-9. DOI: 10 . 1007 / $978-3-540-$ 44502-9

675 [2] A. Calvo, K. Schlueter, E. Tejado, et al. "Self-passivating tungsten alloys of the system W-Cr-Y for high temperature applications". In: International Journal of Refractory Metals and 735 Hard Materials 73 (2018), pp. 29-37. Dor: 10 . $1016 / \mathrm{j}$. i jrmhm.2018.01.018

[3] J. W. Coenen, S. Antusch, et al. "Materials for DEMO and reactor applications-boundary conditions and new concepts".

口In: Physica Scripta T167 (2016), p. 014002. DoI: 10.1088/ 740 0031-8949/2016/T167/014002
[4] S. Cui, Russ P. Doerner, et al. "Thermal conductivity degradation and recovery in ion beam damaged tungsten at different temperature". In: Journal of Nuclear Materials 511 (2018), pp. 141-147. Dor: $10.1016 / \mathrm{j} \cdot$ jnucmat.2018.09.002

[5] R. P. Doerner, A. Grossman, et al. "Response of beryllium to deuterium plasma bombardment". In: Journal of Nuclear Materials 257.1 (1998), pp. 51-58. Dor: 10 . 1016/S00223115(98)00435-8

[6] W. Eckstein. Computer Simulation of Ion-Solid Interactions. Vol. 10. Springer Series in Materials Science. Berlin and Heidelberg: Springer, 1991. ISBN: 978-3-642-73515-8. Dor: 10 . 1007/978-3-642-73513-4

[7] W. Eckstein, Garcia-Rosales C., et al. "Sputtering Data: IPPReport 9/82”. In: (1993).

[8] A. Eksaeva, E. Marenkov, et al. "ERO modelling of tungsten erosion in the linear plasma device PSI-2". In: Nuclear Materials and Energy 12 (2017), pp. 253-260. Dor: 10 .1016/j . nme.2017.03.014

[9] R. G. Faulkner, S. Song, et al. "A model describing neutron irradiation-induced segregation to grain boundaries in dilute alloys". In: Metallurgical and Materials Transactions A 27.11 (1996), pp. 3381-3390. Dor: 10.1007/BF02595431

[10] G. Federici, C. Bachmann, et al. "DEMO design activity in Europe: Progress and updates". In: Fusion Engineering and Design 136 (2018), pp. 729-741. Dor: $10.1016 / \mathrm{j}$.fusengdes . 2018.04 .001

[11] M.I Guseva, A.L Suvorov, et al. "Sputtering of beryllium, tungsten, tungsten oxide and mixed $\mathrm{W}-\mathrm{C}$ layers by deuterium ions in the near-threshold energy range". In: Journal of $\mathrm{Nu}$ clear Materials 266-269 (1999), pp. 222-227. Dor: 10.1016/ S0022-3115(98)00819-8

[12] A. Hasegawa, M. Fukuda, et al. "Neutron irradiation effects on the microstructural development of tungsten and tungsten alloys". In: Journal of Nuclear Materials 471 (2016), pp. 175183. Dor: $10.1016 / \mathrm{j} \cdot$ jnucmat.2015.10.047

[13] Y. Hirooka, M. Bourham, et al. "Evaluation of tungsten as a plasma-facing material for steady state magnetic fusion devices". In: Journal of Nuclear Materials 196-198 (1992), pp. 149-158. Dor: 10.1016/S0022-3115(06)80022-X

[14] Y. Igitkhanov, B. Bazylev, et al. Design Strategy for the PFC in DEMO Reactor (KIT Scientific Reports ; 7637). 2013. DoI: 10.5445/KSP/1000032116

[15] ITER. "Material Specification for the Supply of Tungsten Plates for the ITER Divertor IDM No.ITERD2EDZJ4". In: ITER Document ().

[16] I. Ivanova-Stanik, M. Poradziński, et al. "Analysis of the optimum impurity mix for the EU DEMO scenario". In: Fusion Engineering and Design 136 (2018), pp. 1313-1316. Dor: 10 . 1016/j.fusengdes.2018.04.130

[17] Y. Kamiura, K. Umezawa, et al. "Characterization of Polycrystalline Tungsten Surfaces Irradiated with Nitrogen Ions by X-ray Photoelectron Spectroscopy". In: MATERIALS TRANSACTIONS 57.9 (2016), pp. 1609-1614. Dor: 10 . $2320 /$ matertrans.M2016107

[18] A. Kreter, C. Brandt, et al. "Linear Plasma Device PSI-2 for Plasma-Material Interaction Studies". In: Fusion Science and Technology 68.1 (2015), pp. 8-14. Dor: 10 . 13182/FST14906 
[19] E. Lassner and W. Schubert. Tungsten: Properties, Chemistry, 800 Technology of the Element, Alloys, and Chemical Compounds. Boston, MA and s.1.: Springer US, 1999. IsBN: 978-1-46137225-7. Dor: $10.1007 / 978-1-4615-4907-9$

[20] Ch. Linsmeier, M. Rieth, et al. "Development of advanced high heat flux and plasma-facing materials". In: Nuclear Fusion 80 57.9 (2017), p. 092007. Dor: 10.1088/1741-4326/aa6f71

[21] A. Litnovsky, T. Wegener, et al. "New oxidation-resistant tungsten alloys for use in the nuclear fusion reactors". In: Physica Scripta T170 (2017), p. 014012. Dor: 10 . 1088/14024896/aa81f5

[22] D. Maisonnier, I. Cook, et al. "The European power plant conceptual study". In: Fusion Engineering and Design 75-79

755 (2005), pp. 1173-1179. Dor: 10.1016/j.fusengdes. 2005. 06.095

[23] H. Mehrer, ed. Diffusion in Solids: Fundamentals, Methods, 815 Materials, Diffusion-Controlled Processes. Vol. 155. Springer Series in Solid-State Sciences. Berlin Heidelberg: Springer-

760 Verlag GmbH, 2007. IsBN: 978-3-540-71486-6. Dor: 10.1007/ 978-3-540-71488-0

[24] Mutzke, A., Schneider, R., Eckstein, W. and Dohmen, R. 820 SDTrimSP Version 5.00 (IPP 12/8). Garching: Max-PlanckInstitut für Plasmaphysik. Tech. rep. 2011.

[25] L. Qiao, P. Wang, et al. "Erosion and deuterium retention of CLF-1 steel exposed to deuterium plasma". In: Physica Scripta T170 (2017), p. 014025. Dor: 10.1088/1402-4896/aa8c5c

[26] J. Roth, J. Bohdansky, et al. Data on low energy light ion sputtering: Report IPP 9/26. Max-Planck-Inst. für Plasmaphysik, 1979.

[27] J. Roth, E. Tsitrone, et al. "Recent analysis of key plasma wall interactions issues for ITER". In: Journal of Nuclear Materials 390-391 (2009), pp. 1-9. Dor: $10.1016 / \mathrm{j}$. jnucmat . 2009. 01.037

[28] K. Schmid, V. Rieger, et al. "Comparison of hydrogen retention in W and W/Ta alloys". In: Journal of Nuclear Materials 426.1-3 (2012), pp. 247-253. Dor: 10 . 1016/ j · jnucmat . 2012.04 .003

[29] J. Schmitz, A. Litnovsky, et al. "Argon-seeded plasma exposure and oxidation performance of tungsten-chromiumyttrium smart alloys". In: Tungsten 455.1-3 (2019), p. 277. Dor: $10.1007 / \mathrm{s} 42864-019-00016-7$

[30] J. Schmitz, A. Litnovsky, et al. "WCrY smart alloys as advanced plasma-facing materials - Exposure to steady-state pure deuterium plasmas in PSI-2". In: Nuclear Materials and Energy (2018). Dor: $10.1016 / \mathrm{j} . \mathrm{nme} .2018 .05 .002$

[31] Tatyana Sizyuk and Ahmed Hassanein. "Effect of surface segregation and mobility on erosion of plasma-facing materials in magnetic fusion systems". In: Journal of Nuclear Materials 458 (2015), pp. 312-318. Dor: 10.1016/j · jnucmat . 2014. 12.105

[32] K. Sugiyama, M. Balden, et al. "Erosion of EUROFER steel by mass-selected deuterium ion bombardment". In: Nuclear Materials and Energy 16 (2018), pp. 114-122. Dor: 10.1016/ 795 j.nme.2018.05.021

[33] K. Sugiyama, K. Schmid, et al. "Sputtering of iron, chromium and tungsten by energetic deuterium ion bombardment". In: Nuclear Materials and Energy 8 (2016), pp. 1-7. Dor: 10 . 1016/j.nme.2016.05.016
[34] M. Z. Tokar. "An assessment for the erosion rate of DEMO first wall”. In: Nuclear Fusion 58.1 (2018), p. 016016. Dor: 10.1088/1741-4326/aa92dd

[35] U. von Toussaint, A. Mutzke, et al. "Simulation of coupled sputter-diffusion effects". In: Physica Scripta T167 (2016), p. 014023. Dor: 10.1088/0031-8949/T167/1/014023

[36] T. Wegener, F. Klein, et al. "Development of yttriumcontaining self-passivating tungsten alloys for future fusion power plants". In: Nuclear Materials and Energy 9 (2016), pp. 394-398. Dor: $10.1016 / \mathrm{j} . \mathrm{nme}$.2016.07.011

10 [37] R. Wenninger, R. Albanese, et al. "The DEMO wall load challenge”. In: Nuclear Fusion 57.4 (2017), p. 046002. Dor: 10 . 1088/1741-4326/aa4fb4

[38] R. Wenninger, R. Kembleton, et al. "The physics and technology basis entering European system code studies for DEMO". In: Nuclear Fusion 57.1 (2017), p. 016011. Dor: 10 . 1088/ 0029-5515/57/1/016011

[39] X. Yang and A. Hassanein. "Atomic scale calculations of tungsten surface binding energy and beryllium-induced tungsten sputtering". In: Applied Surface Science 293 (2014), pp. 187190. Dor: $10.1016 / \mathrm{j}$. apsusc. 2013.12.129

[40] Y. Zayachuk, M.H.J. 't Hoen, et al. "Deuterium retention in tungsten and tungsten-tantalum alloys exposed to highflux deuterium plasmas". In: Nuclear Fusion 52.10 (2012), p. 103021. Dor: $10.1088 / 0029-5515 / 52 / 10 / 103021$ 